\title{
STUDY OF THE SOLVENT EXTRACTION OF THE LIGHTER LANTHANIDE METAL IONS BY MEANS OF ORGANOPHOSPHORUS EXTRACTANTS
}

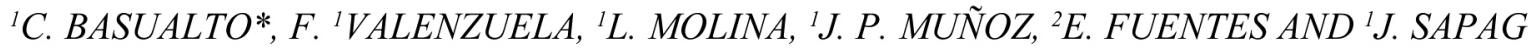 \\ 'Laboratorio de Operaciones Unitarias e Hidrometalurgia, Facultad Ciencias Químicas y Farmacéuticas. Universidad de Chile, Santos Dumont 964, \\ Independencia, Santiago, Chile. \\ ${ }^{2}$ Facultad de Ciencias Quimicas y Farmacéuticas, Universidad de Chile, Sergio Livingstone Polhammer 1007, Casilla 233, Santiago, Chile
}

(Received: January 31, 2013 - Accepted: April 5, 2013)

\begin{abstract}
In recent years there has been a great demand for rare earth metals because of their unique physical and chemical properties as new constituents for electronic, optical and magnetic devices. Because of their extremely similar chemical and physical properties, it is very difficult to separate them. A study of solvent extraction of lighter lanthanide metal ions with a family of organophosphorus extractants, commercially known as D2EPHA, PC88A, CYANEX 272 and CYANEX 301, was made. In general it was found that praseodymium ions were extracted with all the extractants, while in decreasing order neodymium, cerium and lanthanum were extracted less efficiently. Moreover, the D2EHPA and PC88A showed higher extraction efficiencies over the whole $\mathrm{pH}$ range used, but they did not allow an effective separation of REE ions. Furthermore, with CYANEX 301 some degree of separation of the metal ions was achieved, but the extraction efficiencies were comparatively lower. On the contrary, using the CYANEX 272 extractant, medium efficiencies of extraction were achieved, but with a lower ability to separate the REE ions.
\end{abstract}

\section{INTRODUCTION}

Group IIIB of the periodic table of the elements is made up of the metallic elements known collectively as rare earths (REE). In general, they present extremely similar chemical and physical properties, and therefore they are very difficult to separate. Within the rare earth group, the elements scandium, yttrium, and lanthanum differ in their atomic structure from the elements cerium to lutetium (the lanthanoids or lanthanides). The rare earth elements always occur in nature in association with one other. Therefore, isolation of the individual elements or groups of them requires costly separation and fractionation processes.

In recent years there has been great demand for rare earth metals because of their unique physical and chemical properties as new constituents in electronic, optical and magnetic devices.

Commercially available rare earth materials have been used as additives in steels or other alloys, permanent magnets, hydrogen storage materials, magnetooptical storage discs, electro- or cathode ray luminescence, etc. Moreover, they have found some possible applications as semiconductor materials. Electric cars may get to use one kilogram of neodymium in their electric engine and ten kilograms of lanthanum in their nickel metal hydride battery [1]. It has been found that the $\mathrm{Yb}$ layer of the $\mathrm{Al} / \mathrm{Yb} / \mathrm{GaAs}$ structure can control the Schottky barrier height [2]. Eu has been used as a material for forming the clouding layer of a double-hetero structure Pb-Eu-Se-Te infrared laser diode [3]. These types of application are expected to progress in the near future and obviously require commercially available ultra-high purity rare earth materials [4]. The most recent applications still under investigation are the following: pyrophoric alloys which ignite in air when struck at an angle [5-6], improving the corrosion resistance of iron and steel; improving the properties of non-ferrous metals and alloys, lanthanide additions have been very effective in enhancing the mechanical properties and increasing the fatigue life of high strength aluminum alloys [7]; in galvanizing applications, to increase toughness and strength and greatly improve corrosion resistance [8-9]; large capture cross-sections for thermal neutrons in nuclear applications, they are often included in control rods to regulate reactor operation [10-11]. One of the most important applications of the rare earth elements is in supermagnetic and superconductor materials, usually permanent magnets based on gadolinium-cobalt, samarium-cobalt or neodymiumiron-boron, with other metals in smaller proportions. Cobalt alloys offer the highest permanent magnet performance known. Use of these materials continues to grow rapidly [12-16].

The rare earth minerals are widespread and plentiful and we should think about the growing demand that there will be for many years to come. Technology now under development will result in needed cost reductions for many rare earth products and will promote the emergence of many new markets.

There are two sources for REE recovery: mineral deposits and the recycling of electric, electronic and other waste materials. Indeed, the accumulation of industrial, electronic and optical wastes has been highlighted as a valuable second source of rare earth metals. For example, the number of scrapped TV-sets has increased year by year to reach several billions in all countries. Rare earth metals are the main components of fluorescent materials, which are contained in TV-tubes. The composition by weight of the fluorescent material waste in a used TV-tube is $23.8 \%$ $\mathrm{Y}, 1.2 \%, \mathrm{Eu}, 38.4 \% \mathrm{Zn}$ and trace amounts of $\mathrm{Fe}, \mathrm{Co}, \mathrm{Ni}, \mathrm{Al}, \mathrm{Cu}$ and $\mathrm{Pb}$ [17]. In 2005 Jiang et al. developed a hydrometallurgical process to recover lanthanum, yttrium and gadolinium from spent optical glass [18]. Wider use of REEs will require both lower-cost refining and purification methods and the development of many new applications that have been held back by the purity issue [19].

Solvent extraction has been applied practically for the separation of rare earth metals [20-21]. The family of extractants obtained as by-products of phosphoric, phosphonic, and phosphinic acids seems to be the most promising in achieving the extraction of rare earth metal ions from aqueous solutions by means of solvent extraction [22-25].

This paper aims to contribute to knowledge on the separation of the lighter lanthanide ions of lanthanum, cerium, praseodymium and neodymium by means of solvent extraction technique using an organophosphorus family of extractants.

\section{EXPERIMENTAL}

The extractants di-2-ethylhexyl phosphoric acid (D2EHPA) Merck Co., 2-ethylhexyl phosphoric acid mono-2-ethylhexyl ester (PC 88A) DAIHACHI Chemical, bis(2,4,4-trimethylpentyl) phosphinic acid (CYANEX 272) and bis(2,4,4-trimethylpentyl)dithiophosphinic acid (CYANEX 301) were used without further purification. CYANEX reagents were provided by CYTEC Chile.

All lanthanide reagents were analytical grade from Merck, and their aqueous solutions were prepared in the concentration range of $0.1-20 \mathrm{mM}$ from $\mathrm{La}\left(\mathrm{NO}_{3}\right)_{3} \cdot 6 \mathrm{H}_{2} \mathrm{O}, \mathrm{Ce}\left(\mathrm{NO}_{3}\right)_{3} \cdot 6 \mathrm{H}_{2} \mathrm{O}, \mathrm{Pr}\left(\mathrm{NO}_{3}\right)_{3} \cdot 6 \mathrm{H}_{2} \mathrm{O}$ and $\mathrm{Nd}_{2} \mathrm{O}_{3}$, at different $\mathrm{pH}$ values measured by an OAKLON model ION700 pH meter using $\mathrm{NaOH}$ or $\mathrm{HCl}$. The organic solutions were prepared at concentrations of $0.1-120 \mathrm{mM}$ of the respective extractant dissolved in kerosene. The kerosene solvent was provided by Shell Chile and was used without further purification.

The experiments were performed by contacting equal $25 \mathrm{~mL}$ volumes of aqueous and organic solutions in an appropriate Erlenmeyer flask, covered and agitated in a shaker for $24 \mathrm{~h}$ until equilibrium was reached. Later, the raffinate 
and loaded organic phases were separated and small samples were taken from the remaining aqueous solution. The analyses of the concentrations of rare earth elements were made by inductively coupled plasma with a mass spectrometry detector Agilent model 700 .

\section{RESULTS AND DISCUSSION}

Theory

The results expressed as the extraction percentage $(\mathrm{E} \%)$ and the distribution coefficient $\left(D_{M}\right)$ were obtained using Equations 1 and 2, respectively.

$$
\begin{aligned}
& \mathrm{E}(\%)=\left(\frac{\left[\mathrm{M}^{3+}\right]_{0}-\left[\mathrm{M}^{3+}\right]_{\mathrm{eq}}}{\left[\mathrm{M}^{3+}\right]_{0}}\right) \\
& \mathrm{D}_{\mathrm{M}}=\left(\frac{\left[\mathrm{M}^{3+}\right]_{0}-\left[\mathrm{M}^{3+}\right]_{\mathrm{eq}}}{\left[\mathrm{M}^{3+}\right]_{\mathrm{eq}}}\right)
\end{aligned}
$$

In both cases, $\left[\mathrm{M}^{3+}\right]_{0}$ and $\left[\mathrm{M}^{3+}\right]_{\text {e }}$ correspond to the concentrations in the feed and raffinate aqueous solutions, respectively. Many authors have reported that the generic reaction stoichiometry for lanthanide elements with organophosphrus extractants (HR) is given by Equation 3 [26-27].

$$
\mathrm{M}^{3+}+\mathrm{m} \overline{(\mathrm{HR})_{2}} \rightleftharpoons \overline{\mathrm{MR}_{3}(\mathrm{HR})_{2 \mathrm{~m}-3}}+3 \mathrm{H}^{+}
$$

where $\mathrm{M}^{3+}$ is the lanthanide metal ion to be extracted from the feed solution, $\mathrm{HR}$ is the extractant contained in the organic phase represented as the dimer $(\mathrm{HR})_{2}$, and $\mathrm{MR}_{3}(\mathrm{HR})_{2 \mathrm{~m}-3}$ is the metal complex produced, soluble only in the organic phase. The overline denotes species in the organic phase. The associated equilibrium constant for the heterogeneous reaction is given by Equation 4 .

$$
\mathrm{K}_{\mathrm{eq}}=\frac{\left[\overline{\left.\mathrm{MR}_{3}(\mathrm{HR})_{2 \mathrm{~m}-3}\right]} \cdot\left[\mathrm{H}^{+}\right]^{3}\right.}{\left[\mathrm{M}^{3+}\right] \cdot\left[\overline{(\mathrm{HR})_{2}}\right]^{\mathrm{m}}}
$$

Thus, the equilibrium constant can be obtained from the linearization of equation 4.

$$
\log \left(\mathrm{D}_{\mathrm{M}} \cdot\left[\mathrm{H}^{+}\right]_{\mathrm{eq}}^{3}\right)=\log \mathrm{K}_{\mathrm{eq}}+\mathrm{m} \cdot \log \left[\overline{(\mathrm{HR})_{2}}\right]_{\mathrm{eq}}
$$

Additionally, a measurement parameter which accounts for the effectiveness of a separation process is the separation factor " $\beta$ ", which is based on the distribution coefficients of the individual metal ions, as shown in Equation 6.

$$
\beta_{M_{1} / M_{2}}=\frac{D_{M_{1}}}{D_{M_{2}}}
$$

\section{Chemical Speciation of REE}

The speciation curves for REE in aqueous phase as a function of $\mathrm{pH}$ must be considered in order to understand the extraction behavior by the SX methodology. The curves were obtained by means of a computational program based on equilibrium constants in aqueous solution considering sulfuric acid and sodium hydroxide for acid and alkaline media, respectively. Experimentally, the aqueous solutions were prepared with the corresponding lanthanide salt, and all of the ions were included in the modeling. The curves were obtained considering $50 \mathrm{mg} / \mathrm{L}$ of total lanthanide ion concentration.

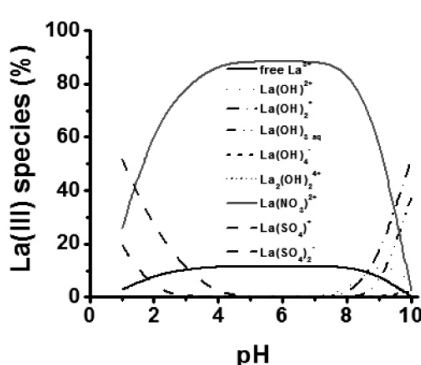

(a)

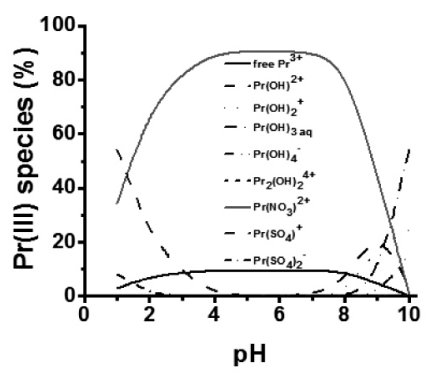

(c)

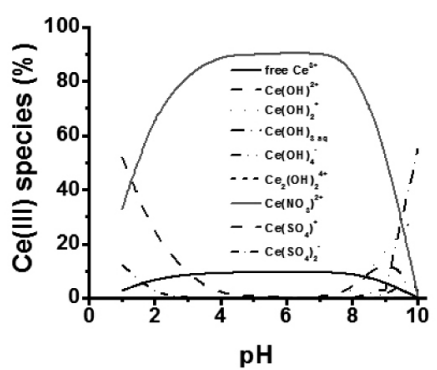

(b)

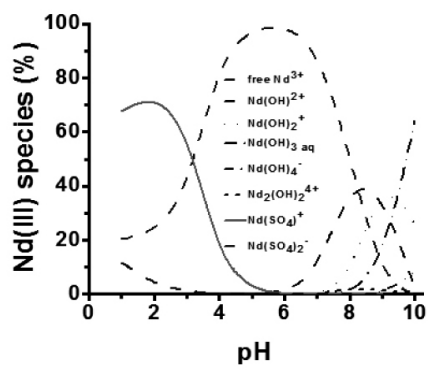

(d)
Figure 1. Curves of chemical speciation in aqueous solution as a function of $\mathrm{pH}$ for:(a) lanthanum, (b) cerium, (c) praseodymium, and (d) neodymium.

In general, the plots show that the REE nitrate or the free REE species predominate largely between approximately $\mathrm{pH} 2$ and $\mathrm{pH} 8-9$. It is also known that the ligand strength of the nitrate species is too weak, so in most cases the REE nitrate species can also be considered as a free REE species.

\section{Extraction of REE by means of organophosphorus extractants}

The optimization of the SX process involves achieving the best extraction conditions in which the uptake of metal ion occurs to the largest extent. To accomplish this goal, this study considered the extraction behavior of the lanthanide elements lanthanum, cerium, praseodymium and neodymium with the organophosphorus extractants D2EHPA, PC88A, CYANEX 272 and CYANEX 301. The effect of the variables that mostly affect the REE extraction process, namely extractant concentration in the organic phase and metal ion concentration in the aqueous feed solution, was studied. In all cases kerosene was used as the organic phase diluent. As extraction measurement parameter, the metal removal percentage $(\mathrm{E} \%)$ and the metal distribution coefficient $\left(\mathrm{D}_{\mathrm{M}}\right)$ -or its $\operatorname{logarithm}\left(\log \mathrm{D}_{\mathrm{M}}\right)$ - were used.

\section{- The effect of extractant concentration in the organic phase}

The effect of varying extractant concentration in the organic phase between 0.96 and $40 \mathrm{mM}$ on the extraction extent of each lanthanide metal ion was studied. In all these experiments, the initial lanthanide metal ion concentration was the same at a constant value of $1 \mathrm{mM}$. In a similar way, the initial $\mathrm{pH}$ of feed solution in all experimental runs was adjusted at a value of 5 . The results are shown in Figure 2.

In Figure 2 it is seen that, as expected, the extraction percentage increases at higher concentrations of the respective extractant. However, at $40 \mathrm{mM}$ of the D2EHPA extractant (Figure 2(a)) all the REEs are extracted around 100\%, which means that their separation is not achieved. In Figure 2(b) it is seen that for a $40 \mathrm{mM}$ concentration of PC $88 \mathrm{~A}$, extractions around 70 and $90 \%$ were reached for the four lanthanide metal ions. A different behavior was seen with the CYANEX 272 and CYANEX 301 extractants: for a $40 \mathrm{mM}$ content of CYANEX 272, Figure 2(c) shows $100 \%$ praseodymium extraction and $20 \%$ lanthanum uptake, while CYANEX 301 in Figure 2(d) achieves close to $75 \%$ praseodymium extraction but less than $10 \%$ lanthanum extraction.

It is known that in the order of lanthanum, cerium, praseodymium, and neodymium, whose atomic numbers are $57,58,59$, and 60 , respectively, the lanthanide contraction of their $3+$ ions occurs. This implies that the ionic ra- 
dius is reduced with increasing atomic number, which in terms of hard soft acid-base theory (HSBA) would consider REE ions as hard acids whose order of hardness would be $\mathrm{Nd}^{3+}>\mathrm{Pr}^{3+}>\mathrm{Ce}^{3+}>\mathrm{La}^{3+}$. Under these same terms, the extractants are considered as hard bases whose hardness decreases in the order D2EHPA $>$ PC88A $>$ CYANEX 272>CYANEX 301 . The analysis of the results, from this point of view, would indicate that the extractant D2EHPA is the hardest base and it would therefore react to a large degree with the four lanthanide ions without discriminating among of them (Figure 2 (a)). As the hardness decreases from PC88A through CYANEX 301, the extent extraction for these REEs would also decrease, but presenting greater discrimination between the different lanthanide metal ions, as can be seen in Figures 2(b), (c) and (d).

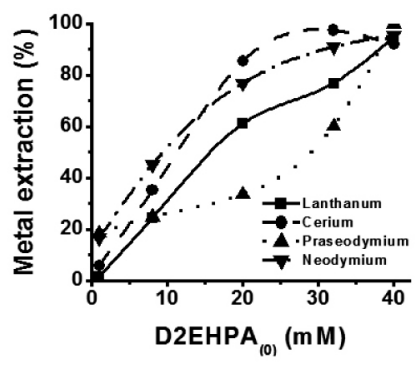

(a)

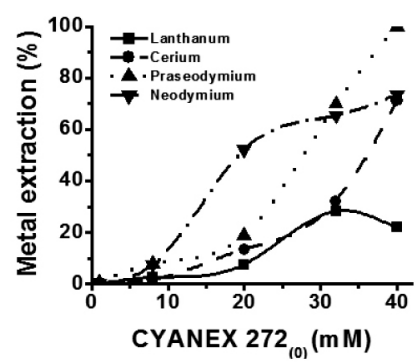

(c)

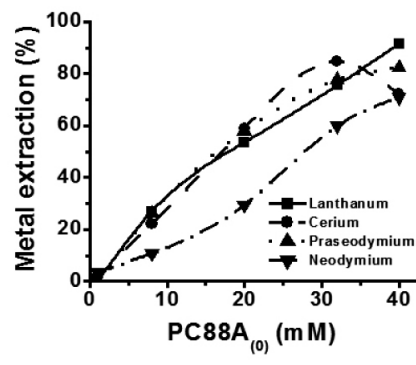

(b)

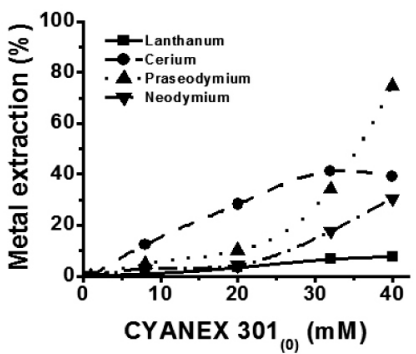

(d)
Figure 2. Extraction behavior of lanthanum, cerium, praseoymium and neodymium at an initial $\mathrm{pH} 5$ with: (a) D2EHPA, (b) PC 88A, (c) CYANEX 272, and (d) CYANEX 301.

-Effect of $\mathrm{pH}$ of the aqueous solution feed

The effect of initial acidity of the aqueous phase on the degree of extraction of REE ions was studied. The experiments were performed varying the initial $\mathrm{pH}$ of the aqueous solution between 1 and 5, keeping the initial concentrations of the REE ions at $1 \mathrm{mM}$. In all the experimental runs the extractant concentration in the organic phase was kept constant at $40 \mathrm{mM}$. The results obtained are shown in Figure 3.

Figure 3 shows that, in general, the extraction percentage of each REE element rises when the initial $\mathrm{pH}$ of the aqueous feed solution increases. Figure 3(a) shows that between $90 \%$ and $100 \%$ metal ion extraction was achieved when D2EHPA was used as extractant. At initial $\mathrm{pH} 5$ complete extraction of all the metals was achieved, and it is not possible to separate them under these conditions. A similar behavior was found for the extraction of lanthanide metals with PC88A, with extraction varying between $70 \%$ and $90 \%$, as seen in Figure 3(b). However, when the extractants CYANEX 272 and CYANEX 301 were used, although extraction increased with increasing initial $\mathrm{pH}$, it is interesting that some degree of separation of the different metal ions was achieved, as seen clearly in Figure 3(c) and Figure 3(d). Since both CYANEX extractants do not show exactly the same behavior for extracting the REE ions, a separation process of these elements can be proposed using the following appropriate extraction sequence: using extractant CYANEX 272 is possible separate neodymium from the other elements around $\mathrm{pH} 4-5$ and a employing a not so high concentration of this extractant, ideally around $20 \mathrm{mM}$. As can be seen in Figure 2 and 3, only neodymium can be well extracted. In a second step, from the raffinate of this first extraction, using CYANEX 301 would be possible to separate praseodymium from lanthanum and cerium around $\mathrm{pH} 5$ and using a higher content of the extractant at the organic phase, around $40 \mathrm{mM}$, given that lanthanum and cerium are much lesser extracted under these experimental conditions. Finally the separation of cerium and lanthanum could be achieved during the stripping step, point which is under study now.

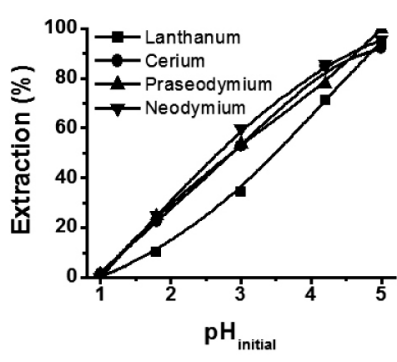

(a)

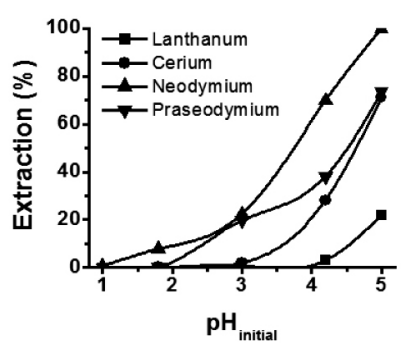

(c)

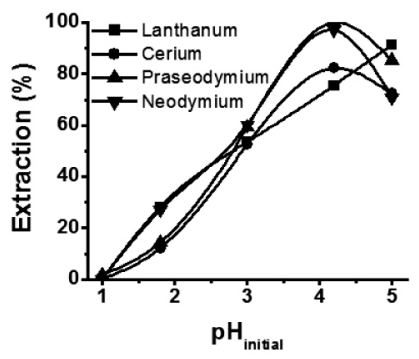

(b)

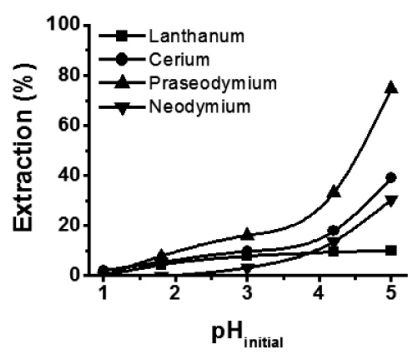

(d)
Figure 3. Effect of initial $\mathrm{pH}$ on the extraction percentage of the REE ions. (a) D2EHPA, (b) PC88A, (c) CYANEX 272, and (d) CYANEX 301.

-Effect of initial metal ion concentration in the aqueous phase

The effect of initial ion concentration in the aqueous phase in the range of 0.16 to $2 \mathrm{mM}$ on the extraction of REE metal ions was studied, keeping the $\mathrm{pH}$ constant at 5. The results are presented in Figure 4.

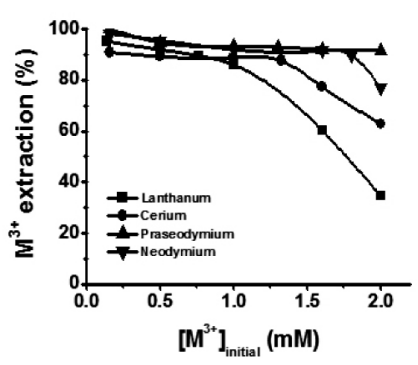

(a)

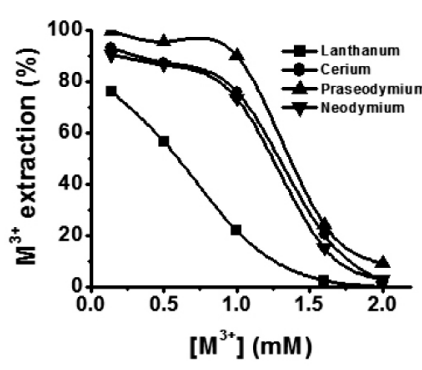

(c)

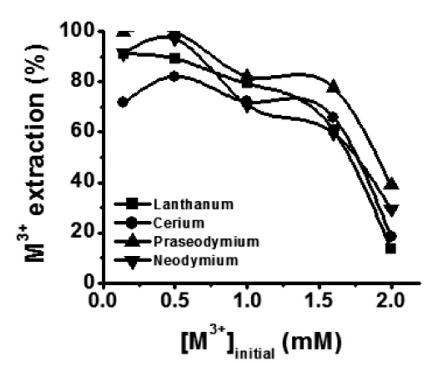

(b)

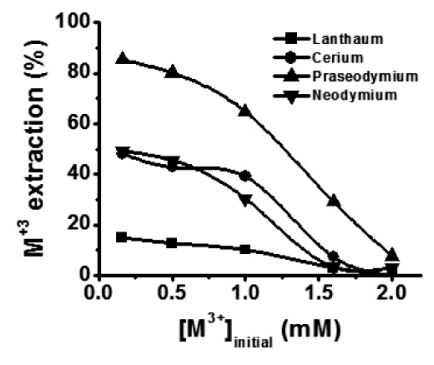

(d)
Figure 4. Effect of the initial concentration of REE ions in the aqueous phase on their extraction percentage: (a) D2EHPA, (b) PC88A, (c) CYANEX 272, and (d) CYANEX 301. 
Figure 4 shows that in all cases the extraction of metals decreases as the initial metal ion concentration increases in the feed solution. Praseodymium was the best extracted REE and lanthanum the one least extracted. It is clear that for higher metal content in the aqueous phase, a higher concentration of extractant in the organic phase is required. These plots are useful to determine the maximum metal loading capacity of each extractant.

\section{-Analysis of the Extraction Equilibrium}

From the experimental data it is possible to obtain additional information, such as the extraction percentages, the distribution coefficients, and the apparent extraction equilibrium constants, and the proposed stoichiometry of the extraction reaction can also be confirmed. To get this information it is necessary to arrange the experimental information and plot it as $\log \left(\mathrm{D}_{\mathrm{M}} \cdot\left[\mathrm{H}^{+}\right]_{\mathrm{eq}}{ }^{3}\right)$ vs. $\log \left(\left[(\mathrm{HX})_{2}\right]_{\mathrm{eg}}\right)$, as shown in equation 5 . Using the slope-method, if the stoichiometries of the extraction reaction follow that proposed as most probable in equation 3, straight lines should be obtained. The slopes of the straight lines would verify the proposed stoichiometry, and from the intercepts of the plots the corresponding apparent equilibrium constants can be obtained. The results are shown in Figure 5.

In Figure 5 it is seen that in all cases straight lines were obtained, confirming that the proposed equations would effectively represent the chemical reactions of the extraction process.

Table 1 summarizes the results of the apparent equilibrium constants $\left(\mathrm{K}_{\mathrm{eq}}\right.$, M) and slopes (m) for all the extraction experiments performed.

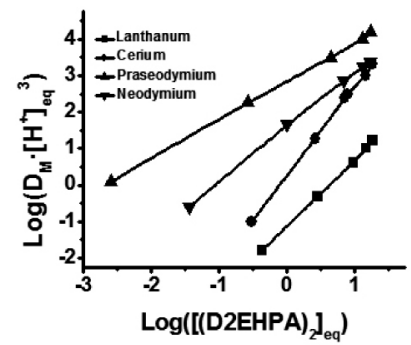

(a)

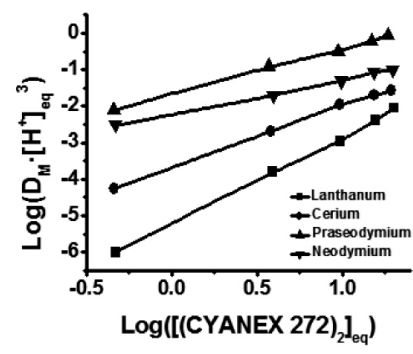

(c)

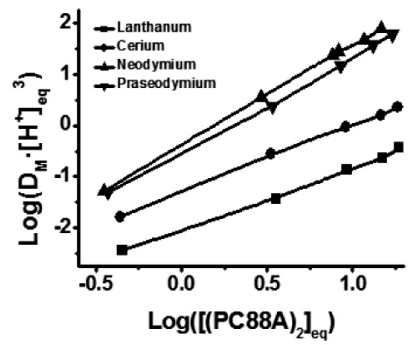

(b)

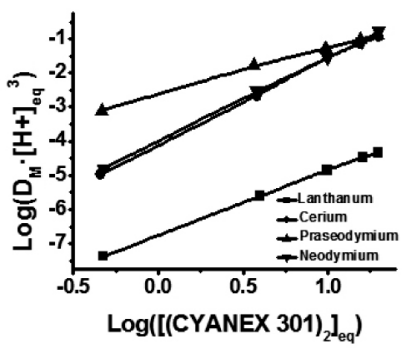

(d)

Figure 5. Log-log graphs of $\mathrm{D}_{\mathrm{M}} \cdot\left[\mathrm{H}^{+}\right]_{\mathrm{eq}}{ }^{3}$ vs. $\left[(\mathrm{HX})_{2}\right]$.

Table 1. Slopes $(\mathrm{m})$ and apparent equilibrium constants $\left(\mathrm{K}_{\mathrm{eq}}\right)$ for the extractions.

\begin{tabular}{|c|c|c|c|c|c|c|c|c|}
\hline & \multicolumn{3}{|c}{ D2EHPA } & \multicolumn{2}{c|}{ PC88A } & \multicolumn{2}{c|}{ CYANEX 272 } & \multicolumn{2}{c|}{ CYANEX 301 } \\
& $\mathrm{m}$ & $\mathrm{K}_{\mathrm{eq}}$ & $\mathrm{m}$ & $\mathrm{K}_{\mathrm{eq}}$ & $\mathrm{m}$ & $\mathrm{K}_{\mathrm{cq}}$ & $\mathrm{m}$ & $\mathrm{K}_{\mathrm{cq}}$ \\
\hline Lanthanum & 1.82 & $7.35 \cdot 10^{-02}$ & 1.22 & $8.96 \cdot 10^{-03}$ & 2.38 & $5.94 \cdot 10^{-06}$ & 1.89 & $1.74 \cdot 10^{-07}$ \\
\hline Cerium & 2.42 & 1.81 & 1.31 & $5.06 \cdot 10^{-02}$ & 1.67 & $2.13 \cdot 10^{-04}$ & 2.52 & $7.60 \cdot 10^{-05}$ \\
\hline Praseodymium & 1.05 & 689 & 1.96 & $4.20 \cdot 10^{-01}$ & 1.25 & $2.15 \cdot 10^{-02}$ & 1.36 & $2.41 \cdot 10^{-03}$ \\
\hline Neodymium & 1.49 & 38 & 1.86 & $2.88 \cdot 10^{-01}$ & 0.99 & $5.87 \cdot 10^{-03}$ & 2.46 & $1.00 \cdot 10^{-04}$ \\
\hline
\end{tabular}

The results indicate that the number of extractant molecules that coordinate in the metal-extractant complex is lower than the expected value of 3 , and their lack would be made up by water molecules to complete the coordination sphere. Furthermore, the variability in the apparent extraction equilibrium constants obtained is higher, but it is clearly seen that praseodymium extraction is the most favored in all cases, followed in decreasing order by neodymium, cerium and lanthanum. Additionally, the results shown in Table 1 indicate that of all the tested extractants, the phosphoric compound D2EHPA would present the most favorable reaction, followed in decreasing order by PC88A, CYANEX 272 and CYANEX 301.

\section{-Separation of REE ions}

A series of experiments in order to measure the degree of extraction and separation of lanthanide metal ions using CYANEX 272 as extractant were made. The experiments were performed varying extractant concentration in the organic phase in the range of 20 to $120 \mathrm{mM}$, using aqueous metal ion solutions whose initial concentration was kept constant at values close to $10 \mathrm{mM}$, with the feed solution at $\mathrm{pH} 4$. The metal distribution coefficients were calculated using equation 2 and the results are plotted in Figure 6.

In Figure 6 it is seen that the distribution coefficients for each metal ion increase to a maximum around a CYANEX 272 concentration of $80 \mathrm{mM}$ in the organic phase. However, it is seen that when the extractant content in the organic phase is increased over that value, the distribution coefficient values of all the metals decrease slightly. This behavior could be explained based on the solubility of the extractant or its complex with the lanthanides ions in the organic phase. In fact, it was observed a certain turbidity in the organic phase what indicates that a grade of insolubility of the metal-extractant complex is attained, when a higher concentration of extractant was used affecting and diminishing the extraction distribution coefficient. In order to define a possible separation route of these elements, the separation factors $\left(\beta_{\mathrm{MI} M 2}\right)$ were calculated using equation 6 . These separation factors allow make it possible to quantify how much of a metal ion is extracted with respect to another one. Based on the results shown in Figure 6, the calculated values of the separation factors are reported in Table 2.

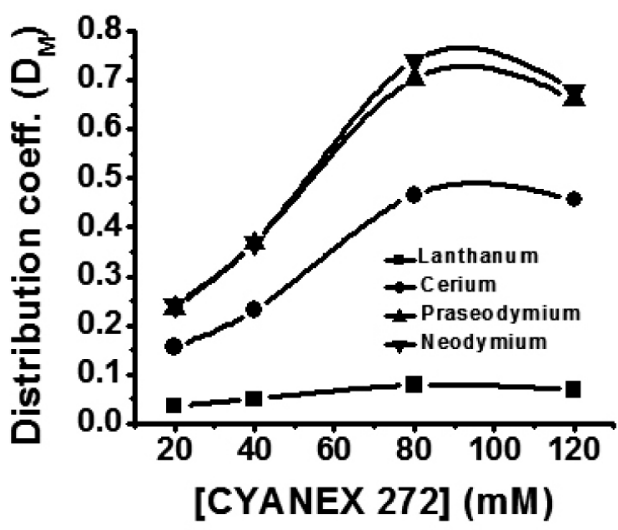

Figure 6. Distribution coefficients of REE as a function of CYANEX 272 concentration. 
Table 2. Separation factors of the light lanthanide ions.

\begin{tabular}{|c|c|c|c|c|c|c|}
\hline \multirow{2}{*}{$\begin{array}{l}\text { CYANEX 272 } \\
\mathrm{mmol} / \mathrm{L}\end{array}$} & \multicolumn{6}{|c|}{ Separation factor $(\beta)$} \\
\cline { 2 - 7 } & $\mathrm{Ce} / \mathrm{La}$ & $\mathrm{Nd} / \mathrm{La}$ & $\operatorname{Pr} / \mathrm{La}$ & $\mathrm{Nd} / \mathrm{Ce}$ & $\mathrm{Pr} / \mathrm{Ce}$ & $\operatorname{Pr} / \mathrm{Nd}$ \\
\hline 20 & 4.3 & 6.5 & 6.7 & 1.5 & 1.6 & 1.0 \\
\hline 40 & 4.6 & 7.4 & 7.4 & 1.5 & 1.6 & 1.0 \\
\hline 80 & 6.0 & 9.0 & 9.5 & 1.5 & 1.6 & 1.1 \\
\hline 120 & 6.7 & 9.7 & 9.9 & 1.5 & 1.5 & 1.0 \\
\hline
\end{tabular}

It is clear that the separation of two or more metal ions will be more feasible as the separation factor $\beta$ reaches values greater than 1.0. Table 2 shows that based on these separation factors, lanthanum ion is the easiest to separate from the other ions. On the contrary, cerium, praseodymium and neodymium are difficult to separate from one another because their selectivity coefficients are all around 1.0.

\section{CONCLUSIONS}

Speciation modeling of REEs in aqueous solution established that these ions occur practically as free ions in the required working range of $\mathrm{pH} 1$ to 5 .

In general, praseodymium metal ion is better extracted with all the extractants studied, followed in decreasing order by neodymium, cerium and lanthanum. Moreover, the extractants D2EHPA and PC88A showed higher extraction efficiencies over the working $\mathrm{pH}$ range, but they do not allow an effective separation of REE ions.

Extractant CYANEX 301 achieves some separation of the metal ions, but its extraction efficiencies are comparatively low, while extractant CYANEX 272 achieved medium extraction efficiencies but showed less ability to separate the REE ions. An appropriate combination of the extractant and working conditions would allow a separation path for lanthanum, cerium, praseodymium and neodymium metal ions contained in a leaching aqueous solution to be established.

\section{ACKNOWLEDGEMENTS}

The financial support of FONDECYT under Project 1100201 is gratefully acknowledged.

Supply of the CYANEX extractants by CYTEC-Chile is appreciated.

\section{REFERENCES}

1.- R. F. Service, Science 327, 1596, (2010)

2.- $\quad$ L. Chang, H. Wang, Y. Cheng, T. Shong, E. Kuang Lin, J. Crystal Growth 198/199, 1092, (1999)

3.- P. Amedzake, E. Brown, U. Hömmerich, S.B. Trivedi, J.M. Zavada, J. Crystal Growth 310, 2015, (2008)

4.- I. Minoru, Vacuum 64, 885, (1996)

5.- Z. Xiaoqin, W. Qudong, L. Yizhen, Z. Yanping, D. Wenjiang, Z. Yunhu, J. Mat. Process Tech. 112, 1, 3, 17, (2001)

6.- X. Q. Zeng, Q. D. Wang, Y. Z. Lü, W. J. Ding, C. Lu, Y. P. Zhu,. C. Q. Zhai, X. P. Xu, Scripta Mat. 43, 5, 14, 403, (2000)

7.- T. Laser, Ch. Hartig, M.R. Nürnberg, D. Letzig, R. Bormann, Acta Materialia 56, 12, 2791, (2008)

8.- $\quad$ Li X., Deng S., Fu H., Mu G., Zhao N. Applied Surface Science 254, 17, 5574, (2008)

9.- H.Wang, R. Akid, Corrosion Sci. 50, 4, 1142, (2008)

10.- J. Kennedy, S. Granville, A. Markwitz, B.J. Ruck, H.J. Trodahl, Nucl Instruments and Methods in Phys Res Section B: Beam Interactions with Materials and Atoms 266, 8, 1558, (2008)

11.- K. Kawano, H. Tasaki, B.-C. Hong, T. Ishitsuka, J. Alloys \& Comp. 451, 1-2, 314, (2008)

12.- T.Saito, J. Magnetism \& Magnetic Mat. 320, 13, 1893, (2008)

13.- W. Kaszuwara, B. Michalski, J. Latuch, M. Leonowicz, J. Magnetism \& Magnetic Mat. 320, 14, e51, (2008)

14.- W.Y. Zhang, M. Stoica, J. Eckert, P. Yu, J.Z. Jiang, Intermetallics 16, 3, 341, (2008)

15.- S.B. Han, J.Y. Lv, X.F. Liu, J. Peng, D.F. Chen, J.H. Li, Z.B. Hu, J.Alloys \& Comp. 431, 1-2, 4, 68, (2007)
16.- H. Fujimoto, H. Shimada, S. Yoshizawa, Physica C: Superconductivity 463-465, 1, 374, (2007)

17.- F. Kubota, K. Shinohara, K. Shimojo, T. Oshima, M. Goto, S. Furusaki, T. Hano, Sep. \& Purification Tech. 24, 93, (2001)

18.- Y. Jiang, A. Shibayama, K. Liu, T., Fujita, Hydrometallurgy 76, 1, (2005)

19.- http://www.arris-intl.com/customer/Papers/remetalspaper.html. Rare Earth Metals Find Interesting New Uses Despite Lack of Engineering Data.By Roman Lundin and John R. Wilson

20.- V. K. Jain, S. G. Pillap, H. C. Mandal, J.Chil. Chem. Soc. 52, 2, 1177 , (2007)

21.- B. N. Kokare, A. M. Mandhare, M. A. Anuse, J. Chil. Chem. Soc. 55 , 4, 431, (2010)

22.- A. A. Abdeltawab, S. Nii, F. Kawaizumi, K. Takahashi, Sep. \& Purification Tech. 26, 265, (2002)

23.- W. Weiwei, W. Xianghan, M. Shulan, L. Hongfei, L. Deqian, J. Rare Earths 24, 685, (2006)

24.- J. Yin, B. Hu, M. He, Z. Jiang, Analytica Chim. Acta 594, 1, 61, (2007)

25.- H. Li, F. Guo, Z. Zhang, D. Z. Wang Li. J. \& Alloys and Comp. 408-412, 995 (2006)

26.- H. Kao, P. Yen y R. Juang, Chem. Eng. J.119, 167, (2006)

27.- G. Seop, M. Uchikoshi, K.Mimura, M. Isshiki, Sep. \& Purification Tech. 67, 79, (2009) 